\title{
Les leviers du transfert des acquis en formation continue du médecin généraliste. Elaboration d'un modèle conceptuel opérationnalisable
}

\author{
Key factors in the transfer of training in continuing medical education \\ of general practicionners. Development of a conceptual operational model
}

\author{
Valérie HUBENS $^{1,2}$, Isabel RAEMDONCK ${ }^{3}$, Geneviève BRUWIER ${ }^{4,5}$, Christelle DEVOS ${ }^{3}$ \\ ${ }^{1}$ Promo santé et médecine générale, Belgique \\ ${ }^{2}$ Fédération des maisons médicales, Belgique \\ ${ }^{3}$ Faculté de psychologie et des sciences de l'éducation, Université catholique de Louvain, Belgique \\ ${ }^{4}$ Société scientifique de médecine générale, Belgique \\ ${ }^{5}$ Département de médecine générale, Université de Liège, Belgique
}

Manuscrit reçu le 4 mars 2016 ; commentaires éditoriaux formulés aux auteurs le 12 septembre 2016 ; accepté pour publication le 29 mai 2017

Mots-clés
Formation continue ;
Médecine Générale ;
Transfert des acquis de
formation ; Théorie ;
du comportement
planifié ; Intention ;
Attitude ; Sentiment
d'efficacité
personnelle ;
Dispositif de
formation ; Etude
longitudinale

Résumé - Contexte : Formation continue en médecine générale en Belgique francophone. But : Identification des leviers du transfert des acquis en formation continue en médecine générale et élaboration d'un modèle conceptuel opérationnalisable reprenant ces facteurs. Méthodes : Etude quantitative longitudinale. Questionnaires remis en fin d'ateliers et six à huit semaines plus tard. Le premier questionnaire (leviers du transfert) a été complété par 187 participants (97\% de taux de réponse), dont 81 ont également complété le second questionnaire de manière valide de 6 à 10 semaines plus tard (transfert auto-rapporté). Résultats : Quatre leviers du transfert ont été identifiés?: l'intention de transférer, l'attitude envers le transfert, le sentiment d'efficacité personnelle et l'orientation de la formation vers le transfert. L'attitude envers le transfert est le facteur explicatif le plus important de l'intention de transférer et le levier ayant l'effet indirect le plus fort sur le transfert. Conclusions : Une version initiale d'un modèle conceptuel opérationnalisable du transfert en formation continue en médecine générale a été élaborée. L'attitude envers le transfert est le facteur clé du transfert en formation continue en médecine générale sur lequel les formateurs peuvent agir, notamment en orientant leur formation vers la transfert. 


Keywords
Coninuous training;
General Practice;
Transfer of training;
Theory of Planned
Behaviour; Intention;
Attitude; Self-effi-
cacy; Training
scheme; Longitudi-
nal Study

Abstract - Context: Continuing medical education of general practicioners in Frenchspeaking Belgium. Aims: Identifying key factors in the transfer of training in continuing medical education of general practicioners and developing a conceptual operational model that take these key factors into account. Methods: Quantitative longitudinal study. Questionnaires were submitted at the end of the workshops and 6 to 8 weeks later. The first questionnaire (key factors in the transfer of training) was filled out by 187 participants (answer rate: $97 \%$ ). There were 81 valid answers in the second questionnaire (self-reported transfer of training). Results: Four key factors were identified: transfer intention, attitude towards transfer, self-efficacy and transfer schemes. Attitude towards transfer is the key factor with the greatest effect on transfer intention and the main indirect factor on transfer. Findings: An intial version of a conceptual operational model of the transfer of training in continuing medical education for general practicioners was developed. Attitude towards transfer is the key factor in continuing medical education of general practicionners that trainers can influence, especially the use of transfer schemes.

\section{Introduction et problématique}

La médecine est en évolution constante. Les médecins généralistes sont amenés à se former de manière continue. Le transfert des acquis de formation constitue un véritable enjeu de la formation continue professionnelle. Chercheurs et praticiens s'accordent à dire que la proportion des apprentissages réalisés en formation qui est ensuite mise en pratique sur le lieu de tavail est faible ${ }^{[1]}$. Les mêmes constats sont faits en formation médicale continue ${ }^{[2,3]}$. Il est donc important d'en comprendre les leviers pour pouvoir l'augmenter. L'objectif de cette recherche est de proposer un modèle conceptuel opérationnalisable, qui rende compte des leviers du transfert des acquis d'apprentissage en formation continue du médecin généraliste. Par le terme « leviers », nous entendons les facteurs ayant un effet sur le transfert et sur lesquels les formateurs ou organisateurs de formation en médecine générale sont en mesure d'avoir une influence. Le transfert des acquis de formation peut être défini comme «le degré de mise en pratique des compétences et des comportements appris dans le contexte du travail $»^{[4]}$, ou, plus précisément dans le cadre de cette recherche, comme l'application des apprentissages dans la pratique médicale, lorsqu'il s'agit, par exemple, de prescrire des examens biologiques (dépistage du diabète de type 2, pathologies thyrö̈diennes, évaluation du risque cardio-vasculaire) selon les recommandations, d'améliorer les performances de la prescription d'imagerie tout en minimisant l'irradiation des patients, ou encore de proposer les dépistages des cancers les plus pertinents aux patients.

Le transfert des acquis de formation a été étudié aussi bien en psychologie des apprentissages ${ }^{[5,6]}$ qu'en psychologie des organisations. En psychologie des organisations, depuis Baldwin et Ford ${ }^{[4]}$, les facteurs influençant les transfert des acquis de formation sont classiquement regroupés en trois catégories : les facteurs individuels, les facteurs liés au dispositif de formation et ceux liés à l'environnement de travail.

Dans un premier temps, une recherche de la littérature en psychologie des organisations a été réalisée pour identifier les leviers susceptibles d'entrer dans le modèle. Ont ensuite été retenus ceux sur lesquels les formateurs ou organisateurs de formation étaient suceptibles de pouvoir agir.

De nombreux auteurs se sont intéressés aux facteurs susceptibles de favoriser le transfert ${ }^{[1]}$. Grossman et Salas ${ }^{[7]}$ ont synthétisé les facteurs ayant montré les liens les plus forts et les plus constants avec le transfert. Parmi les facteurs individuels, sont identifiés la capacité cognitive, le sentiment d'efficacité personnelle (SEP), la motivation et l'utilité perçue. Parmi les facteurs liés au dispositif de formation, les pratiques suivantes ont montré des liens forts avec les transfert : donner la formation dans un 
environnement aussi proche que possible de celui dans lequel les compétences acquises seront utlisées, informer à propos des erreurs potentielles et de la manière de les gérer, donner des explications claires sur les comportements à apprendre, démontrer leur utilisation efficace, mettre en avant les opportunités de les mettre en pratique, réaliser du feedback et mettre en place un renforcement. En Belgique francophone, Devos et al. ${ }^{[8]}$ ont, par ailleurs, trouvé que l'orientation de la formation vers le transfert, à savoir le degré auquel la formation est pensée pour rendre les participants capables de transférer leur apprentissage dans le milieu du travail, explique $15.2 \%$ de la variance du transfert. Parmi les facteurs liés à l'environnement de travail, Grossman et Salas ${ }^{[7]}$ ont identifié : a) le climat de transfert et sa dimension sociale ; b) le soutien par les responsables et par les pairs ; c) l'opportunité de transférer et : d) le suivi, à savoir les feedbacks postformation ou encore la mise à disposition d'outils facilitant le transfert tels que des aides de type informationnel, procédural ou des aides à la décision.

Parmi l'ensemble de ces facteurs, nous avons identifié quatre leviers sur lesquels il était possible d'agir en tant que formateur : le dispositif de formation, la motivation à transférer, le SEP en fin de formation et l'utilité perçue. La transformation de l'environnement de travail des médecins généralistes n'est pas, a priori, accessible aux formateurs ou organisateurs de formation.

Ces quatre leviers sont repris dans le modèle du transfert de Gegenfurtner et al. ${ }^{[9]}$ qui ont effectué de nombreux travaux dans le domaine. Ce modèle positionne la motivation à transférer comme variable médiatrice entre le dispositif de formation, le SEP et l'utilité perçue, d'une part, et le transfert des acquis de la formation, d'autre part. Ces quatre leviers sont également pris en compte dans la théorie du comportement planifié de Ajzen ${ }^{[10]}$. Cette théorie a été suggérée dans le cadre de l'étude du transfert des acquis de formation par Cheng et Hampson ${ }^{[11]}$ dans la mesure où les apprenants ont la possibilité de choisir de transférer ou non, et/ou ils peuvent choisir ce qu'ils transfèrent. Ce cadre théorique a ainsi été choisi car les médecins généralistes jouissent d'une grande autonomie et parce qu'il s'agit d'une théorie du changement de comportement robuste et validée ${ }^{[10]}$. Par ailleurs, la revue systématique de Légaré $^{[3]}$ et al. de 76 études ayant utilisé les théories socio-cognitives pour expliquer les comportements cliniques des professionnels de la santé a montré que cette théorie était la plus appropriée pour prédire le comportement des professionnels de santé.

La théorie du comportement planifiée ${ }^{[10]}$ nous permet de porter un nouveau regard sur le transfert des acquis de formation. Outre la présence des quatre leviers identifiés dans la littérature, cette théorie suggère deux nouveaux leviers potentiels, à savoir : a) la contrôlabilité perçue par rapport au transfert, définie comme la perception par l'individu du degré auquel un comportement dépend de lui et : b) la perception des normes, qui renvoie à la pression sociale pour mettre en œuvre ou non le comportement. Nous avons ainsi retenu, pour notre étude, six leviers potentiels pour le transfert des acquis de formation continue en médecine générale : l'attitude envers le transfert (comme variable pour refléter l'utilité perçue du transfert), le SEP par rapport au transfert, l'orientation de la formation vers le transfert (comme variable pour représenter le dispositif de formation ${ }^{[8]}$ ), l'intention de transférer (comme variable pour appréhender la motivation à transférer), la perception des normes par rapport au transfert et la contrôlabilité perçue par rapport au transfert.

Parmi ces six leviers, les quatre permiers ont déjà montré des liens forts et constants avec le transfert ${ }^{[7]}$, tandis que les deux suivants sont théoriques, issus de la théorie du comportement planifié de Ajzen ${ }^{[10]}$.

Par ailleurs, trois de ces facteurs font partie des quatre facteurs identifiés par Légaré et al. ${ }^{[3]}$ dans le cadre du développement d'un outil théorique pour évaluer l'impact du développement professionnel continu (dont la formation continue) sur les intentions de mettre en pratique le nouveau comportement clinique. Ces facteurs sont les croyances à propos des capacités (facteur proche de celui du SEP), les croyances à propos des conséquences (facteur proche de l'attitude envers le transfert) et l'infuence sociale (facteur proche de la perception des normes par rapport au transfert). 


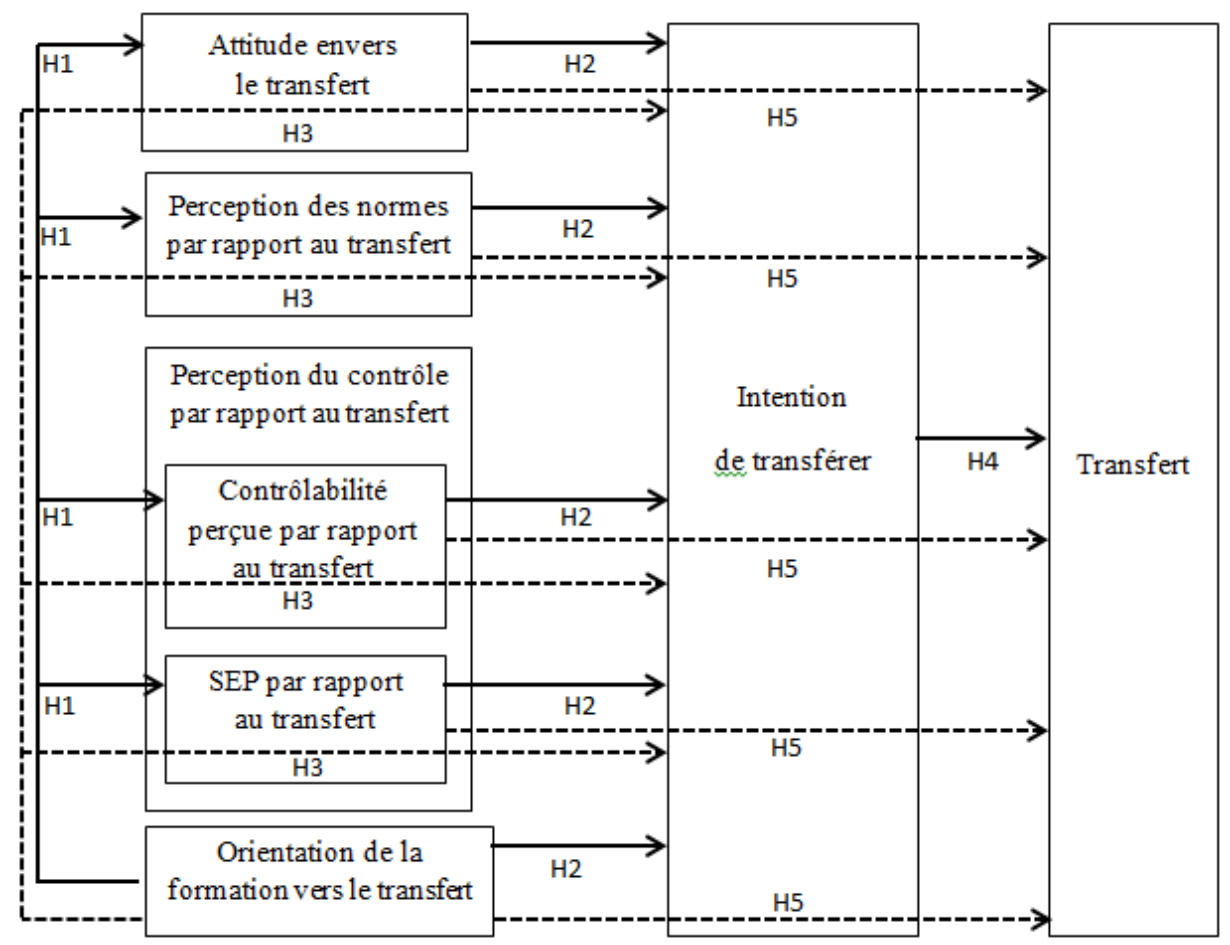

$\longrightarrow$ Hypothèses de prédiction

SEP : Sentiment d'efficacité personnelle

Fig. 1. Modèle et hypothèses sur les leviers potentiels du transfert des acquis en formation continue en médecine générale.

\section{Question et hypothèses de recherche}

La question de recherche est : «quels sont les leviers du transfert en formation continue en médecine générale et comment peuvent-ils être modélisés ». L'articulation des leviers identifiés dans la littérature sur la base de la théorie du comportement planifié de Ajzen ${ }^{[10]}$ et en lien avec le modèle du transfert de Gegenfurtner et al. ${ }^{[9]}$, ont amené à l'élaboration de cinq hypothèses modélisées dans la figure 1. Cette étude empirique vise à examiner systématiquement la viabilité de ces cinq hypothèses, de manière à commencer à documenter la validité de construit d'une version initiale de ce modèle.
Le modèle de Gegenfurtner et al. ${ }^{[9]}$ positionne le dispositif de formation, outre comme antécédent de la motivation à transférer, comme antécédent du SEP et de l'utilité perçue. L'utilité perçue fait partie des facteurs influençant l'attitude envers le transfert. Associant ces informations à la théorie du comportement planifié, nous formulons une première hypothèse : l'orientation de la formation vers le transfert prédit l'attitude envers le transfert, la perception des normes par rapport au transfert, la contrôlabilité perçue par rapport au transfert, et le SEP par rapport au transfert.

Selon Ajzen ${ }^{[10]}$, l'intention de mettre en pratique un comportement est prédite par l'attitude, la 
perception des normes par rapport au transfert et la perception du contrôle par rapport à ce comportement. Selon Gegenfurtner et al. ${ }^{[9]}$, la motivation à transférer est également déterminée, entre autres, par les caractéristiques de la formation. Ceci nous amène à formuler l'hypothèse $2:$ l'attitude envers le transfert, la perception des normes par rapport au transfert, la contrôlabilité perçue par rapport au transfert, le SEP par rapport au transfert et l'orientation de la formation vers le transfert prédisent l'intention de transférer.

Le rôle médiateur du SEP et de l'utilité perçue entre le dispositif de formation et la motivation à transférer sont suggérés dans le modèle de Gegenfurtner et al. ${ }^{[9]}$ Transposant ces informations à la théorie du comportement planifié, l'hypothèse 3 est formulée : l'attitude envers le transfert, la perception des normes par rapport au transfert, la contrôlabilité perçue par rapport au transfert et le SEP par rapport au transfert jouent un rôle médiateur entre l'orientation de la formation vers le transfert et l'intention de transférer.

Selon la théorie du comportement planifié ${ }^{[10]}$, l'intention est l'antécédent immédiat du comportement, ce qui en fait un bon prédicteur de ce dernier, laissant présager, comme suggéré par Hutchins et al. ${ }^{[12]}$, la possible utilisation de la mesure de l'intention de transférer comme mesure du transfert. L'hypothèse 4 est ainsi formulée : l'intention de transférer prédit le transfert.

Le rôle médiateur de l'intention ou de la motivation à transférer est implicite dans le modèle de Ajzen ${ }^{[10]}$, et proposé explicitement par Gegenfurtner et al. ${ }^{[9]}$. Ce rôle de médiation a, par ailleurs, été observé par Devos et al. ${ }^{[8]}$ et Peters ${ }^{[13]}$ en ce qui concerne la motivation à transférer. Transposant ces résultats à la théorie du comportement planifié ${ }^{[10]}$, l'hypothèse 5 peut être émise : l'intention de transférer joue un rôle médiateur entre les différents leviers que sont l'attitude envers le transfert, la perception des normes par rapport au transfert, la contrôlabilité perçue par rapport au transfert, le SEP par rapport au transfert, l'orientation de la formation vers le transfert et le transfert.

L'ensemble des hypothèses est modélisé dans la figure 1 .

\section{Méthodes}

Une approche quantitative longitudinale a été choisie. L'approche quantitative permet d' analyser le poids de chaque facteur sur le transfert, et d'étudier précisément les interrelations entre ceux-ci (par exemple, les effets de médiation). Par ailleurs, l'aspect longitudinal permet de clarifier la direction des effets et d'éviter que le degré de transfert n'influence la perception des leviers du transfert.

\section{L'échantillon}

Il s'agit d'un échantillon non probabiliste, de convenance. En effet, l'étude a été réalisée sur huit ateliers interactifs de $1 \mathrm{~h} 30$, programmés dans le cadre d'une semaine de formation continue organisée par la Société scientifique de médecine générale (SSMG), à laquelle les médecins généralistes se sont inscrits de manière volontaire. Les médecins généralistes ont été répartis dans huit groupes différents de manière aléatoire par le secrétariat. Chaque groupe a suivi les huit ateliers dans l'ordre établi dans leur programme. Les médecins généralistes n'ont été interrogés qu'en lien avec le troisième atelier suivi, de sorte que les animateurs étaient bien rôdés. Ont été exclus de l'échantillon les médecins généralistes ayant la majeure partie de leur activité profesionnelle en dehors de la médecine générale.

\section{Mesures}

Le premier questionnaire reprenant les six leviers potentiels du transfert a été remis aux médecins généralistes à la fin de leur atelier (temps 1). Ce questionnaire reprenait 25 items mesurant six variables correspondant aux six leviers potentiels identifiés. Chaque échelle a été construite à partir d'échelles existant dans la littérature et comportant au moins quatre items. La théorie du comportement planifié de Ajzen ${ }^{[10]}$ ne propose pas d'échelles standard mais bien des consignes pour construire sa propre échelle adaptée à son contexte. Les échelles «attitude envers le transfert», " perception des normes par rapport au transfert », « contrôlabilité 
perçue par rapport au transfert » et «SEP par rapport au transfert » ont ainsi été construites en suivant les consignes de Francis et al. ${ }^{[14]}$ et en s'inspirant des items de l'échelle utilisée par Shoham ${ }^{[15]}$ pour la contrôlabilité perçue, et de celles utilisées par Shoham ${ }^{[15]}$ et Devos et al. ${ }^{[8]}$ pour le SEP. L'échelle « orientation de la formation vers le transfert » (transfer design) est issue du Learning Transfer System Inventory (LTSI) traduite en français par Devos et al. ${ }^{[8]}$. L'échelle « intention de transférer » est inspirée de celle de Hutchins et al. ${ }^{[12]}$

Le questionnaire a été pré-testé dans deux versions successives par respectivement quatre et six médecins généralistes, et approuvé par des personnes-ressources dans le cadre du transfert des acquis ou de la formation continue des médecins généralistes.

Le deuxième questionnaire mesurait le transfert. L'échelle de quatre items a été contruite à partir de l'échelle de Devos et al. ${ }^{[8]}$. Le questionnaire a été prétesté par trois médecins généralistes. Un lien vers le questionnaire en ligne (LimeSurvey) a été envoyé par mail six semaines après la fin de la semaine de formation (temps 2). Un rappel a été envoyé dix jours plus tard.

L'anonymat a été assuré aux répondants grâce à l'utilisation d'un code (première lettre du prénom du père, jour de naissance $1-31$, première lettre du prénom de la mère) permettant d'apparier les deux questionnaires.

\section{Les variables}

L'attitude envers le transfert correspond au degré auquel une personne évalue celui-ci favorablement ou non $^{[10]}$. L'échelle finale $(\alpha=0.85)$, suite à l'analyse factorielle en composante principale, contient trois items, par exemple : « Mettre en pratique ce que j'ai appris dans l'atelier a du sens ». Les indices de saturation des items de l'échelle vont de 0.82 à 0.92 .

La perception des normes par rapport au transfert renvoie à la pression sociale pour mettre en oeuvre ou non le transfert ${ }^{[10]}$. L'échelle finale (alpha de Cronbach 0.75) contient trois items, par exemple : « Il est attendu des médecins généralistes qu'ils mettent en pratique ce qu'ils ont appris dans l'atelier ». Les indices de saturation vont de 0.65 à 0.90 .

La contrôlabilité perçue par rapport au transfert est le degré auquel le transfert est perçu par un individu comme dépendant de lui ${ }^{[10]}$. L'échelle finale (alpha de Cronbach 0.75) contient trois items, par exemple : « Mettre en pratique ce que j'ai appris dans l'atelier dépend de ma seule décision ». Les indices de saturation vont de 0.78 à 0.87 .

Le sentiment d'efficacité personnelle (SEP) par rapport au transfert est le degré de confiance que l'individu a en sa capacité à réaliser le transfert ${ }^{[10]}$. L'échelle finale (alpha de Cronbach 0.74) contient les quatre items de départ, par exemple : « Mettre en pratique ce que j'ai appris dans l'atelier me semble pouvoir se faire aisément ». Les indices de saturation vont de 0.68 à 0.87 .

L'orientation de la formation vers le transfert est le degré auquel la formation a été conçue pour donner aux participants la capacité de transférer les acquis sur le lieu du travail (Devos et al. ${ }^{[8]}$ ). L'échelle finale (alpha de Cronbach 0.72) contient les cinq items de départ, par exemple : «Les activités et exercices utilisés par les formateurs m'ont aidé à savoir comment mettre en pratique ce que j'ai appris dans l'atelier ». Les indices de saturation vont de 0.68 à 0.76 .

L'intention de transférer est l'engagement délibéré et déterminé à mettre en pratique les acquis de la formation (adapté de Hutchins et al. ${ }^{[12]}$ ). L'échelle finale (alpha de Cronbach 0.89) contient les quatre items de départ, par exemple : «Je projette de mettre en pratique ce que j'ai appris dans l'atelier ». Les indices de saturation vont de 0.79 à 0.90 .

Le transfert renvoie à l'évaluation auto-rapportée par les participants du degré auquel ils ont mis en pratique des acquis de la formation (adapté de Wexley et Latham, cité par Baldwin \& Ford ${ }^{[4]}$ ). L'échelle finale (alpha de Cronbach 0.78) contient les quatre items de départ, par exemple :«J'ai mis en pratique ce que j'ai appris dans l'atelier dans mon activité professionnelle ». Les indices de saturation vont de 0.76 à 0.84 . 
Analyses

L'ensemble des données ont été encodées et traitées avec le logiciel SPSS. Les items des échelles formulés de manière négative ont été inversés. La normalité de la distribution a été testée pour toutes les variables. Toutes les variables sont distribuées normalement et permettent ainsi d'utiliser les tests paramétriques pour les analyses inférentielles.

Une analyse factorielle (analyse en composante principale) avec rotation varimax a été réalisée sur l'ensemble des échelles. La fidélité de chaque échelle a été mesurée avec le coefficient alpha de Cronbach. Ces deux opérations ont déterminé le nombre d'items maintenus dans chaque échelle. Le coefficient alpha de Cronbach vérifie la dimension « consistance interne » de la fidélité. En éducation, l'analyse factorielle est surtout utilisée dans le but d'évaluer la validité de construit d'instruments psychométriques et c'est ce que documentent les coefficients (ou indices de saturation) ${ }^{[16]}$.

La moyenne a ainsi pu être calculée pour chaque variable. Les corrélations entre toutes les variables ont été mesurées de manière à avoir un aperçu global des liens entre celles-ci.

En ce qui concerne plus spécifiquement le test des différentes hypothèses, les tests statistiques suivants ont été sélectionnés : les hypothèses de prédiction ont été testées par la régression linéaire simple ( $\mathrm{H} 1$ et $\mathrm{H} 4)$ ou la régression linéaire multiple $(\mathrm{H} 2)$ tandis que les hypothèses de médiation $(\mathrm{H} 3$ et $\mathrm{H} 5)$ ont été testées avec l'approche par bootstrap (Hayes ${ }^{[17]}$ ) .

\section{Résultats}

Parmi les 199 médecins généralistes ayant suivi leur troisième atelier, 193 médecins généralistes ont répondu au premier questionnaire juste après la formation, ce qui représente un taux de réponse de $97 \%$. Quatre questionnaires dont les répondants exerçaient essentiellement hors de la médecine générale et deux questionnaires qui étaient fortement incomplets ont été éliminés, de sorte qu'il restait 187 questionnaires valides. L'échantillon est composé de $60.4 \%$ d'hommes et $39 \%$ de femmes. Le nombre moyen d'années de pratique est de 32.4 (SD 8.9). En ce qui concerne le type de pratique, $59.4 \%$ sont des médecins travaillant seuls, $18.2 \%$ travaillent avec au moins un autre médecin et $18.2 \%$ travaillent dans une équipe pluridisciplinaire.

Parmi les 199 médecins ayant suivi le troisième atelier, 144 médecins ont répondu au second questionnaire en ligne. Pour diverses raisons telles que l'incapacité de travail depuis le retour de formation, le questionnaire non suffisamment rempli, les réponses de médecins n'ayant pas rempli le premier questionnaire, les codes incomplets et le manque de discrimination du code permettant d'apparier le premier et le deuxième questionnaire, seuls 81 questionnaires ont été gardés.

Le profil et les réponses au premier questionnaire de ces 81 médecins généralistes ont été comparés aux profil et réponses des 106 autres médecins généralistes ayant répondu uniquement au premier questionnaire, en utilisant le test $\mathrm{du} \mathrm{Chi}^{2}$ pour les variables catégorielles et le test $\mathrm{t}$ de Student pour les variables continues. Aucune différence significative n'a été trouvée pour quelque variable que ce soit. L'ensemble des 187 questionnaires a donc été gardé pour l'analyse des variables du temps 1 .

Dans le tableau de corrélations (tableau I), nous observons d'emblée que le transfert n'est corrélé de manière significative $(p<0.05)$ qu'avec deux autres variables : l'intention de transférer et le SEP par rapport au transfert. Par contre, l'intention de transférer est corrélée de manière très significative $(p<0.01)$ avec les autres variables. La corrélation la plus forte $(r=0.80)$ est celle entre l'attitude envers le transfert et l'intention de transférer. Si l'attitude envers le transfert n'est pas corrélée de manière significative avec le transfert, elle l'est, par contre, de manière très significative $(p<0.01)$ avec les autres variables. Ceci est également le cas pour l'orientation de la formation vers le transfert. Cependant, si le calcul des coefficients de corrélation permet effectivement de documenter un lien entre les variables, il ne permet pas de postuler que ce lien soit un lien de causalité.

L'hypothèse 1 , selon laquelle l'orientation de la formation vers le transfert prédit l'attitude envers le transfert, la perception des normes par rapport au 
Tableau I. Coefficients de corrélation de Pearson entre les différents leviers potentiels du transfert des acquis de formation en médecine générale.

\begin{tabular}{|l|c|c|c|c|c|c|c|}
\cline { 3 - 8 } \multicolumn{2}{l|}{} & Attitude & Normes & Contrôlabilité & SEP & Formation & Intention \\
\hline Attitude & & 1 & & & & & \\
\hline Normes & & $0.29^{* *}$ & 1 & & & & \\
\hline Contrôlabilité & & $0.43^{* *}$ & $0.16^{*}$ & 1 & & & \\
\hline SEP & & $0.38^{* *}$ & 0.08 & $0.41^{* *}$ & 1 & & \\
\hline Formation & & $0.65^{* *}$ & $0.33^{* *}$ & $0.35^{* *}$ & $0.42^{* *}$ & 1 & \\
\hline Intention & & $0.80^{* *}$ & $0.31^{* *}$ & $0.46^{* *}$ & $0.46^{* *}$ & $0.62^{* *}$ & 1 \\
\hline Transfert & & 0.09 & 0.16 & -0.004 & $0.22^{*}$ & 0.11 & $0.28^{*}$ \\
\hline
\end{tabular}

**. La corrélation est significative au niveau 0.01 (bilatéral).

*. La corrélation est significative au niveau 0.05 (bilatéral).

SEP : Sentiment d'efficacité personnelle

transfert, la contrôlabilité perçue par rapport au transfert et le SEP par rapport au transfert, est validée pour l'ensemble des variables. En effet tous les résultats des régressions linéaires simples sont significatifs $(p<0.001)$.

Afin de tester l'hypothèse 2 selon laquelle l'attitude envers le transfert, la perception des normes par rapport au transfert, la contrôlabilité perçue par rapport au transfert, le SEP par rapport au transfert et l'orientation de la formation vers le transfert prédisent l'intention de transférer, une régression linéaire multiple a été réalisée avec l'ensemble des variables.

Le modèle est significatif $(F(5)=79.2, p<$ 0.001 ). Le degré de multi-colinéarité (corrélation entre les variables prédictrices) est acceptable (indice de tolérance $>0.10$, VIF $<10$ ). L'ensemble des variables prédictrices explique $67.8 \%$ de la variance de l'intention.

L'hypothèse 2 est partiellement acceptée. En effet, lorsque tous les prédicteurs sont considérés de manière simultanée, seules les variables " attitude envers le transfert $»(\beta=0.62, \quad p<0.001)$ et $\ll$ SEP par rapport au transfert $»(\beta=0.13, p<0.01)$ restent des prédicteurs significatifs de l'intention de transférer. L'attitude envers le transfert a la plus large contribution par rapport à cette prédiction.

Pour tester l'hypothèse 3 , selon laquelle il y aurait médiation de l'attitude envers le transfert, la perception des normes par rapport au transfert, la contrôlabilité perçue par rapport au transfert et le SEP par rapport au transfert entre l'orientation de la formation vers le transfert et l'intention de transférer, l'approche par bootstrap (Hayes ${ }^{[17]}$ ) a été utilisée.

La médiation est confirmée pour l'attitude envers le transfert (effet 0.44, IC95\% 0.30, 0.57), et le SEP par rapport au transfert (effet 0.06, IC95\% 0.017, 0.114 ).

Afin de vérifier l'hypothèse 4, selon laquelle l'intention de transférer prédit le transfert, une régression linéaire a été effectuée avec l'intention comme variable indépendante et le transfert comme variable dépendante. Comme il n'y a qu'un seul facteur indépendant dans le modèle testé, le coefficient de régression standardisé ( $\beta=0.28, p<0.05$ ) est équivalent au coefficient de régression. Le résultat de la régression montre que l'intention expliquerait $6.5 \%$ de la variance du transfert $(F(1)=6.52, p<0.05)$. L'hypothèse est ainsi confirmée.

Afin de tester l'hypothèse 5 selon laquelle l'intention de transférer joue un rôle médiateur entre l'attitude envers le transfert, la perception des normes par rapport au transfert, la contrôlabilité perçue par rapport au transfert, le SEP par rapport au transfert, l'orientation de la formation vers le transfert et le transfert, l'approche de par bootstrap a également été utilisée (Hayes ${ }^{[17]}$ ).

Etant donné que seuls l'attitude envers le transfert et le SEP par rapport au transfert restaient significatifs 


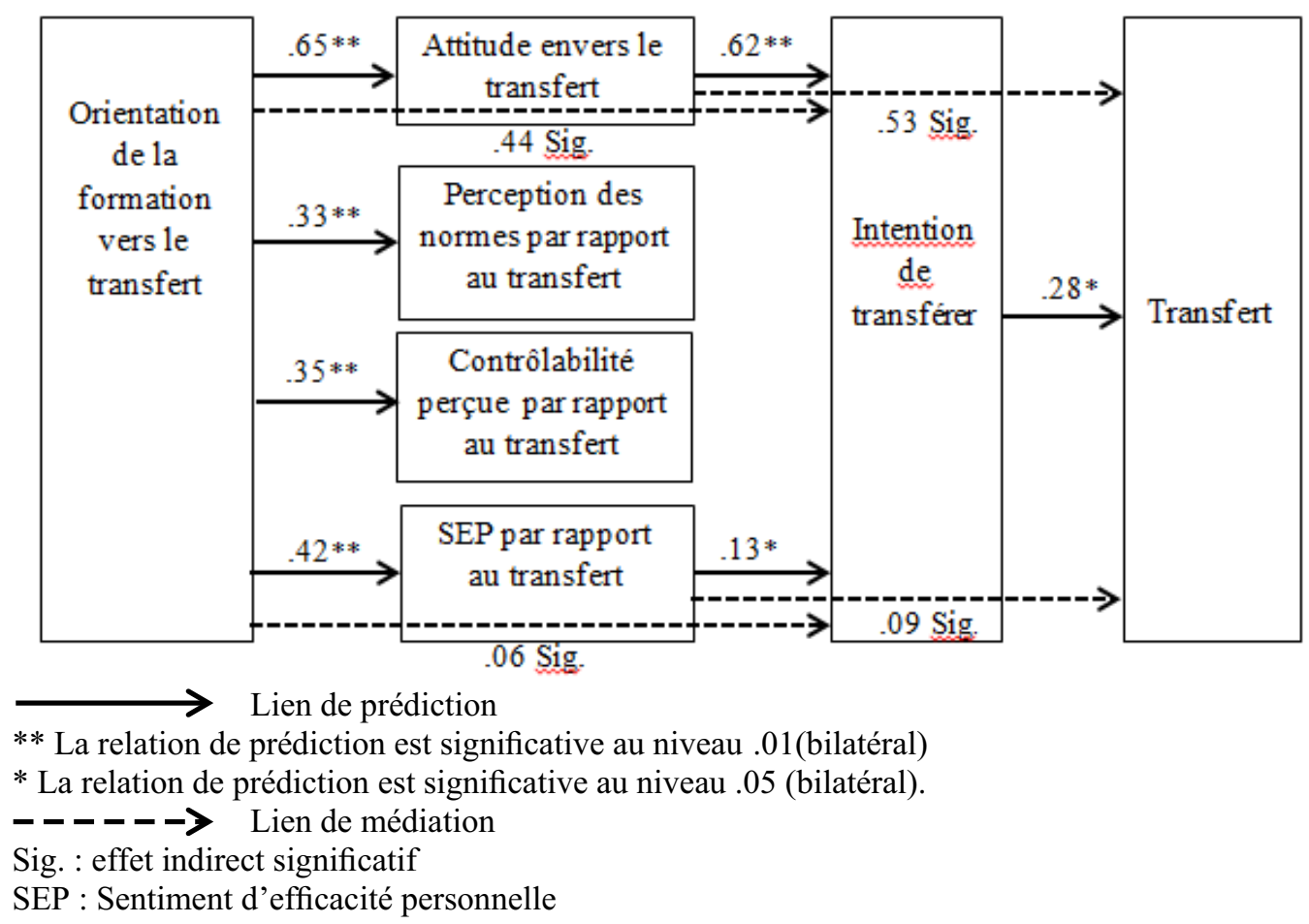

Fig. 2. Modèle des leviers du transfert des acquis en formation continnue en médecine générale avec les hypothèses confirmées.

lorsque tous les prédicteurs étaient intégrés dans le même modèle de régression multiple (cf. hypothèse 2), seuls les effets indirects de ces deux facteurs ont été envisagés. L'effet indirect de l'attitude envers le transfert (effet 0.53 , IC95\% 0.196, 0.846) et celui du SEP par rapport au transfert (effet 0.09, IC95\% 0.006, 0.220) sur le transfert sont significatifs. Il y a donc bien médiation dans les deux cas.

La figure 2 synthétise l'ensemble des hypothèses confirmées.

\section{Discussion}

L'étude met en évidence un levier direct du transfert en formation continue en médecine générale : l'intention de transférer. L'intention de transférer est corrélée positivement $(r=0.28, p<0.05)$ au transfert : plus le médecin généraliste a l'intention de transférer en fin de formation plus il rapporte avoir transféré les acquis de la formation 6 à 10 semaines plus tard. L'intention de transférer explique $6.5 \%$ de la variance du transfert. La théorie du comportement planifié ${ }^{4]}$ laissait présager une corrélation plus forte entre l'intention de transférer et le transfert. Il y a donc probablement de nombreux facteurs d'influence intervenant entre la fin de la formation, moment auquel l'intention de transférer a été mesurée, et le transfert dans la pratique professionnelle. Ces facteurs se situent probablement dans l'environnement de travail, tels que par exemple, la surcharge de travail, la pression du temps, l'utilisation de rappels informatiques, ou encore, pour ceux qui travaillent en équipe, le soutien des collègues et la fixation d'objectifs d'équipe.

Pour chercher à influencer l'intention de transférer, on cherchera à agir avant tout sur l'attitude envers le transfert et également sur le SEP par rapport au transfert. En effet, l'étude a identifié ces deux 
variables comme leviers indirects du transfert qui auront un effet sur celui-ci par le biais de leur effet sur l'intention de transférer. De ces deux leviers indirects identifiés, c'est l'attitude envers le transfert qui semble être la variable clé à la fois du transfert («Effet indirect attitude » $=0.53$, sig. versus « Effet indirect SEP $»=0.09$, sig. $)$ et de l'intention de transférer $(\beta=$ $0.62, p<0.001$ versus $\beta=0.13, p<0.05$ ). L'attitude envers le transfert présente en effet un lien indirect envers le transfert et un lien direct envers l'intention de transférer beaucoup plus forts que ceux du SEP par rapport au transfert.

Pour influencer l'attitude envers le transfert des médecins généralistes, il paraît pertinent pour le formateur de partager avec eux et d'échanger, dans les formations, des informations susceptibles d'influencer positivement leur attitude. Ces informations peuvent être d'ordres différents et varier en fonction du sujet de la formation. Ainsi, peuvent être abordés, par exemple, la plus-value pour la santé du patient, le niveau de preuve scientifique (Evidence Based Medecine), le gain de temps potentiel en consultation, la fréquence d'utilisation en médecine générale, le fait que cela va pouvoir les aider à répondre aux sollicitations des patients suscitées, par exemple, par des campagnes de prévention. Cet échange serait également l'occasion d'entendre les réticences au transfert de la part des médecins généralistes et de chercher à les atténuer.

Prenant en compte l'apport du modèle de Gegenfurtner et al. ${ }^{[9]}$, l'orientation de la formation vers le transfert avait également été envisagée comme prédictrice de l'intention de transférer. Les résultats indiquent que celui-ci jouerait non pas un effet direct sur l'intention de transférer, mais agirait sur cette dernière de manière indirecte par le biais de son effet sur l'attitude envers le transfert et sur le SEP par rapport au transfert.

Ainsi, l'orientation de la formation vers le transfert semble être un levier intéressant pour agir sur les facteurs influençant l'intention de transférer en formation continue en médecine générale, que ce soit l'attitude envers le transfert ou le SEP par rapport à la formation. L'orientation de la formation vers le transfert peut ainsi également être considérée comme un levier indirect du transfert par son effet indirect sur l'intention de transférer. S'assurer que la formation soit orientée vers le transfert se fait, notamment, en citant beaucoup d'exemples de possibilités de mise en pratique, en utilisant des situations similaires à celles rencontrées dans la pratique professionnelle ainsi que des activités et exercices permettant aux médecins généralistes d'anticiper comment mettre le contenu en pratique, de manière à les rendre confiants en leur capacité à transférer. Il est également intéressant d'anticiper, avec les participants, les obstacles à la mise en œuvre et de chercher des pistes pour les dépasser.

\section{Limites de l'étude}

Les limites de cette étude sont liées à la mesure du transfert, au nombre limité de questionnaires valides mesurant le transfert (deuxième questionnaire) et aux analyses statistiques.

En ce qui concerne la mesure du transfert, celleci a été, comme pour l'ensemble des autres variables, réalisée de manière auto-rapportée. Or, bien que la majorité des études mesurent le transfert de cette manière, il est de plus en plus souvent recommandé de multiplier les sources de mesures du transfert en impliquant, par exemple, les collègues et/ou les responsables ${ }^{[13]}$. Ceci n'était pas envisageable dans le cadre de cette étude, les médecins généralistes n'ayant pas de responsable ou de collègues en mesure de vérifier s'ils mettent bien en pratique les acquis de la formation.

Une autre limite de la mesure du transfert est que l'on n'a pas la certitude que les médecins généralistes ne mettaient pas déjà le contenu de la formation en pratique avant celle-ci et qu'il est donc difficile d'affirmer qu'il s'agit bien de la mesure du transfert d'acquis de la formation.

Le taux d'attrition constitue également une limite à cette étude. Le manque de discrimination du code en est la principale raison et pourrait être facilement corrigé dans le cadre de futures études.

Une dernière limite concerne les analyses statistiques. En effet des analyses de prédiction de variables ayant été mesurées au même moment ont été 
réalisées. Ainsi l'orientation de la formation vers le transfert a été considérée comme variable prédictrice de l'attitude envers le transfert, des normes par rapport au transfert, de la contrôlabilité du transfert et du SEP par rapport au transfert, alors que l'ensemble de ces variables se trouvaient toutes sur le même questionnaire, présenté en fin de formation. En cas de réplication de l'étude, il serait intérressant d'envisager des temps différents pour mesurer ces variables.

\section{Perspectives pour les recherches futures}

Le modèle issu de nos résultats (figure 2) pourrait faire l'objet de travaux de validation opérationnelle. Le modèle pourrait ainsi encore être amené à évoluer.

Chercher à influencer positivement l'attitude envers le transfert est la piste principale qui ressort de cette étude pour agir directement sur l'intention de transférer et indirectement sur le transfert. Il a été noté qu'orienter la formation vers le transfert était un moyen de le faire. Il serait cependant intéressant d'investiguer d'autres moyens d'agir sur ce levier. Faisant référence à la théorie du comportement planifié $\left(A j z e{ }^{[10]}\right)$, une première piste serait de réaliser une recherche exploratoire afin d'identifier les croyances partagées par les médecins généralistes qui influencent leur attitude envers le transfert. Pour que les médecins généralistes évaluent positivement un comportement à mettre en pratique, celui-ci doit-il être, à leurs yeux, utile pour le patient, utile pour la santé publique, acceptable pour le patient, d'un rapport coût-bénéfice acceptable, ou encore, représenter un gain de temps,...?

\section{Conclusions}

Une version initiale d'un modèle conceptuel opérationnalisable du transfert en formation continue médicale a été élaboré. Il s'agit maintenant de poursuivre avec des travaux de validation opérationnelle. En fonction des résultats, le modèle pourrait peut-être être amené à évoluer.
Cette étude a tenté par ailleurs de contribuer à l'amélioration de la formation continue en médecine générale, en identifiant les facteurs qui peuvent influencer positivement le transfert et sur lesquels les formateurs et organisateurs de formation peuvent agir. Quatre leviers du transfert ont ainsi été identifiés : l'intention de transférer, l'attitude envers le transfert, le sentiment d'efficacité personnelle (SEP) par rapport au transfert et l'orientation de la formation vers le transfert.

Parmi ceux-ci, le facteur clé est l'attitude du médecin généraliste envers le transfert. Celle-ci est en effet le facteur explicatif le plus important de l'intention de transférer et celui ayant l'effet indirect le plus fort sur le transfert. Une première manière d'avoir un effet positif sur l'attitude envers le transfert est de s'assurer que la formation soit orientée vers le transfert.

\section{Contributions}

Valérie Hubens a mené l'étude dans le cadre d'un mémoire de master en Sciences de l'éducation. Elle a conçu le protocole, effectué le recueil et l'analyse des données, interprété les résultats et rédigé le manuscrit. Christelle Devos a dirigé l'étude et participé à la rédaction du manuscrit. Isabel Raemdonck a co-dirigé l'étude, participé à la révision critique et à l'approbation de la version finale. Geneviève Bruwier a co-dirigé l'étude, participé à la révision critique et à l'approbation de la version finale.

\section{Liens d'intérêts}

Aucune auteure ne déclare de conflit d'intérêt en lien avec le contenu de cet article.

\section{Références}

1. Devos C, Dumay X. Les facteurs qui influencent le transfert : une revue de la littérature. Savoirs, 2006;12:11-46. 
2. Davis D, Thomson O'Brien MA, Freemantle N, Wolf FM, Mazmanian P, Tailors-Vaiscy A. Impact of Formal Continuing Medical Education Do Conferences, Workshops, Rounds, and Other Traditional Continuing Education Activities Change Physician Behavior or Health Care Outcomes? JAMA 1999;282:867-874

3. Légaré F, Borduas F, Freitas A, Jacques A, Godin G, CPD-KT team. Development of a Simple 12-Item Theory-Based Instrument to Assess the Impact of Continuing Professional Development on Clinical Behavioral Intentions. PLoS ONE 2014;9(3): e91013. doi:10.1371/journal.pone.009101

4. Baldwin TT, Ford JK. Transfer of training : A review and directions for future research. Personnel Psychology 1988;41:63-103.

5. Tardif J. Le transfert des apprentissages. Montréal : Les Éditions Logiques, 1999.

6. Frenay M, Bédard D. Le transfert des apprentissages. In : Bourgeois E, Chapelle G. (eds.) Apprendre et faire apprendre. Paris : PUF, 2006:123-35.

7. Grossman, R, Salas, E. The transfer of training : what really matters. International Journal of Training and Development 2011;5:103-20.

8. Devos, C, Dumay, X, Bonami, M, Bates, RA, Holton, EF. The Learning Transfer System Inventory (LTSI) translated into French : internal structure and predictive validity. International Journal of Training and Development 2007;11:181-99.

9. Gegenfurtner, A, Veermans, K, Festner, D, Gruber, H. Motivation to transfer training : An Integrative Literature Review. Human Resource Development Review 2009;8:403-23.

10. Ajzen, I. The Theory of Planned Behavior. Organizational Behavior and Human Decision Processes 1991;50:179-211.
11. Cheng, EWL, Hampson, I. Transfer of training : A review and new insights. International Journal of Management Reviews 2008;10:327-41.

12. Hutchins HM, Nimon K, Bates R, Holton E. Can the LTSI Predict Transfer Performance? Testing intent to transfer as a proximal transfer of training outcome. International Journal of Selection and Assessment 2013;2:251-63.

13. Peters S. Transfert des acquis de formation : Modélisation de l'impact de variables liées à l'apprenant, au design pédagogique, et à l'environnement de travail. Thèse de doctorat en Sciences psychologiques et de l'éducation non publiée. Liège : Université de Liège, 2012.

14. Francis JJ, Eccles MP, Johnston M, Walker A, Grimshaw J, Foy R et al. Constructing questionnaires based on the Theory of Planned Behaviour. A manual for health Services Researchers. Newcatsle upon Tyne : University of Newcastle, 2004.

15. Shoham A. Predicting Trainees Intentions to Transfer Training. An application of the Theory of Planned Behavior. Mémoire de maîtrise en psychologie non publié. Montréal : Université de Montréal, 2008.

16. Bourque J, Poulin N, Cleaver AF. Évaluation de l'utilisation et de la présentation des résultats d'analyses factorielles et d'analyses en composantes principales en éducation. Revue des sciences de l'éducation 2006;32:325-44.

17. Hayes AF. Beyond Baron and Kenny : Statistical Mediation Analysis in the New Millenium. Communication Monographs 2009;76:408-420.

Correspondance et offprints: Valérie Hubens, Fédération des maisons médicales, Boulevard du midi 25/5, 1000 Bruxelles. Mailto : valerie.hubens@fmm.be 